\title{
Rotator Cuff Case Report: Diagnosis and Treatment Using the Biopuncture Protocol
}

\author{
Jan Kersschot* \\ Private Clinic, Antwerp Area, Belgium \\ Submission: December 15, 2016; Published: February 14, 2017 \\ *Corresponding author: Jan Kersschot, Private Clinic, Antwerp Area, Belgium, Email: jan@kersschot.com
}

Keywords: Rotator cuff; Functional diagnosis; Intramuscular injection; Biopuncture

\section{Introduction}

A female patient presented with pain in the right shoulder and limitation of both abduction and internal rotation of the right arm for several months. She received two intra-articular cortisone injections from a first doctor, but she reported that she experienced no improvement. She looked for an approach without corticosteroids and contacted a second doctor. Clinical examination revealed painful points in the deltoid muscle. Ultrasound revealed tendinopathy with calcifications. Her pain, limitation of abduction and internal rotation of the right arm improved after six sessions with intramuscular injections of ultra-low dose medications (ULDMs) in her right deltoid muscle. This technique is also described as Biopuncture [1]. ULDMs are defined as medications containing one or several ingredients which are homeopathically diluted below Avogadro's number.

\section{Patient Information and Presenting Concerns}

Patient is a Caucasian female, born on December 11, 1956, and works in a bank in Belgium as a financial assistant for several decades. Her medical, family and psychosocial history is negative. She complained about pain in the right shoulder and limitation of abduction and internal rotation of the right arm for several months. She cannot recall any specific activities related to the shoulder complaints.

\section{Clinical Findings}

The patient presented with pain in the right shoulder. When the patient was asked to pinpoint the exact location of her pain, she pointed to the anterior and lateral part of the right deltoid muscle. The pain was not referring to the neck or elbow. There was no numbness in the arm or hand. There was no pain during the night. The pain in the deltoid muscle was worse during abduction of the arm. Abduction was limited to about the horizontal level $\left(90^{\circ}\right)$, internal rotation was also limited (not measured). Ultrasound (April 14, 2016) was normal, except for an inflammatory tendinopathy of the supraspinatus tendon, and a few small peri-tubercular calcifications. No lab tests were performed. Clinical examination revealed muscular pain points in the anterior and lateral part of the deltoid muscle. No other clinical tests were performed.

\section{Timeline}

A. First Week of January 2016: Pain in the Right Shoulder (Gradual Onset).

B. February 2016: Intra-articular Joint Injection of Cortisone in the Right Shoulder (the patient reported no clinical improvement after the first injection).

C. March 2016: Intra-articular Joint Injection of Cortisone in the Right Shoulder (the patient reported no clinical improvement after the second injection).

D. March 2016 till May 2016: Six sessions of ULDM injections in the deltoid muscle (the patient reported gradual clinical improvement).

\section{Diagnostic Focus and Assessment}

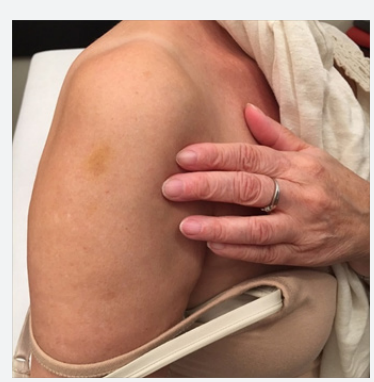

Figure 1: Patient Shows Pain Zone nr 1 (Anterior Part of the Right Deltoid Muscle). 


\section{Journal of Complementary Medicine \& Alternative Healthcare}

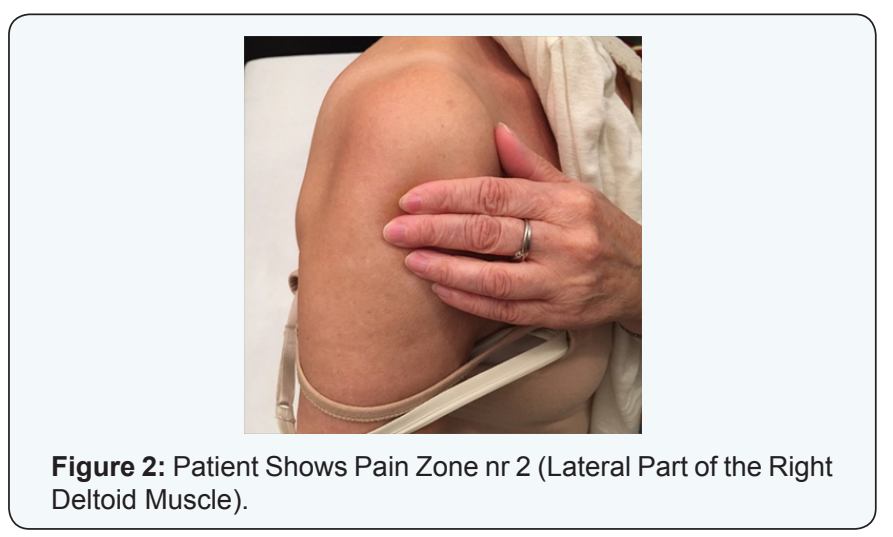

In this patient, the conventional diagnosis (CD), based on the ultrasound, was rotator cuff pathology, with calcifications of the supraspinatus tendon. But the clinical examination was based on the pain zones as identified by the patient (Figure 1\&2). In this zone, a palpation-guided diagnostic approach was performed (Figure 3\&4). In two areas of the deltoid muscle (anterior part and lateral part), muscular pain points were identified. Such muscular pain points can produce several clinical symptoms, as described in Table 1. It was hypothesized that these muscular pain points produced the pain in the shoulder and the restriction of movement in the right arm (functional diagnosis: FD). This hypothesis was tested by applying biopuncture into these two muscular pain points [2]. Remark: The photographs were not taken during the first session; that is why one can notice the blue spot of a previous injection.

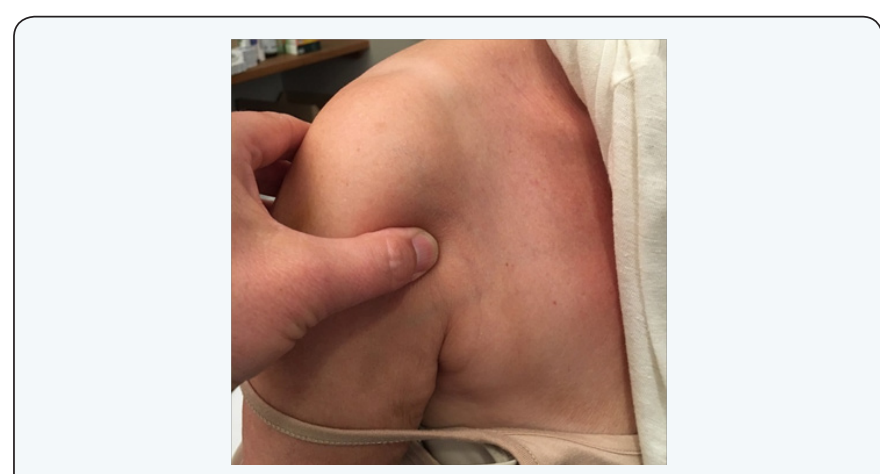

Figure 3: Doctor Examines Pain Zone nr 1 (Anterior Part of the Right Deltoid Muscle).

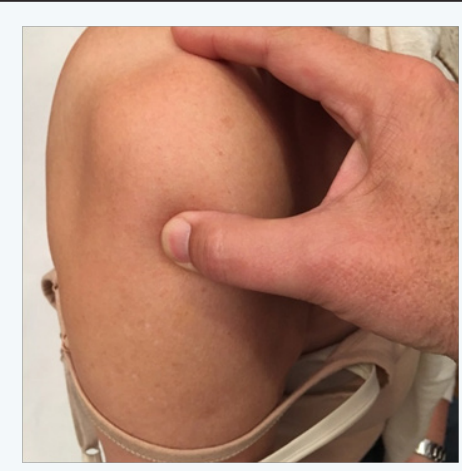

Figure 4: Doctor Examines Pain Zone nr 2 (Lateral Part of the Right Deltoid Muscle).
Table 1: Clinical Picture of the Muscle Dysfunction.

\begin{tabular}{|c|c|}
\hline 1 & Muscular Pain (Local Pain or Referred Pain) \\
\hline 2 & Muscular Weakness \\
\hline 3 & Restriction of Movement (Joint Hypo mobility) \\
\hline 4 & Muscular Stiffness \\
\hline
\end{tabular}

\section{Therapeutic Focus and Assessment}

The patient received local intramuscular injections at a depth of $2 \mathrm{~cm}$ in the deltoid muscle: three injections in the anterior part and three injections in the lateral part of the muscle. In a syringe of $5 \mathrm{~mL}$, the following products were used: $2 \mathrm{~mL}$ of the ULDM and 3mL of sodium chloride. On March 23 and March 29, the ULDM was Myosotis Compositum, on April 5 and April 18 Arnica Compositum was used. On April 25 and May 3, Ferrum Compositum was used

The visual analog scale was used to check the pain during treatment. She had $8 / 10$ on March $23,8 / 10$ on March 29, 7/10 on April 5, 6/10 on April 18, 4/10 on April 25 and 2/10 on May 3. Follow-up by phone confirmed that the pain in the deltoid muscle subsided almost completely, and she cancelled the seventh appointment. There was no opportunity to have a follow-up ultrasound investigation or a follow-up ultrasound clinical examination. She refused CT or MRI scan because of claustrophobic reactions to such environments.

To exclude the influence of other therapeutic interventions, a mono therapy was applied, meaning that apart from the injections no other therapeutic measures (such as oral support with painkillers/NSAIDs or local application of Arnica Compositum) were applied.

\section{Follow-up and Outcomes}

The patient was asked to come for treatment once a week (remark: there was a two weeks' interval after the third session for practical reasons). After the first two sessions, the patient experienced only minor improvement of the pain in the shoulder, but the internal rotation and abduction of the arm were slightly better. The latter was not measured with hard data, but was reported subjectively by the patient (e.g., she mentioned that she was able again to wash her hair with both hands and to put on her bra with both hands on the back). After a series of six sessions, the shoulder pain as well as the internal rotation and abduction of the arm improved substantially.

\section{Discussion}

The conventional approach of rotator cuff is mainly based on clinical examination, X-rays, Ultrasound and MRIa (magnetic resonance imaging). In some cases, a series of three intralesional injections (e.g., intra-articular joint injections or bursa injections) with cortisone are given. Although the side effects are very well known and the long term results disappointing, this 
procedure is still standard today. Instead of cortisone, one could also inject Traumeel [3,4] or Traumeel and Zeel [5] into the joint. But these injections with ULDMs are not always efficient. This lack of clinical outcome (in some cases) is sometimes regarded as a failure of the medication, especially when this has been done in a setting of a clinical study. But one could wonder if the injection with the ULDM was given at the right location. If a shoulder pain has its origin in the deltoid muscle, intra-articular injections with ULDMs will not produce any short or long term effects-apart from the effect of the needle on pain modulation (e.g., gate control).

The patient presented with both shoulder pain on the anterior and lateral part of the deltoid muscle, and limitation of shoulder joint mobility. This clinical picture is very common in general practice, and in some cases the origin of the pain and limited shoulder movement is intra-articular, sometimes peri-articular and sometimes both. Radiological tools may reveal different types of injuries, such as osteoarthritis, joint degeneration, bursitis, supraspinatus tendinopathy (partial tears, tears) and/or calcifications. These findings are then directly correlated to the clinical picture of the patient, hypothesizing that the radiological findings are really relevant for that patient (Hypothesis 1). Nobody will deny the findings by the radiologist, but we know that there are many false positive and false negative radiological findings (see later). False positive findings may lead to unnecessary treatment options (e.g., surgery), and false negative findings may leave the patient with the frustration that nobody seems to find the cause of the pain, and doctors often conclude that the pain is mainly psychogenic and not somatic.

When patients present with shoulder pain with a partial tear of the supraspinatus tendon and/or with calcifications near the supraspinatus tendon, the question remains: Are these radiological findings really the cause of the clinical picture? And what about patients who have pain in the shoulder but who do not have any abnormal findings on X-rays, Ultrasound and MRIa? What if we find pain points in the deltoid muscle, exactly in the area where that particular patient is complaining about? Could we presume that these muscular pain points are the origin of the pain (Hypothesis 2)? The only way to find out is to test this second hypothesis in a particular patient by treating these soft tissue lesions. If one could obtain clinical improvement in that way, without prescribing conventional pain killers or NSAIDs, one could conclude that the second hypothesis may be valid.

These days, physicians tend to rely more and more on technical support by medical imagery and less and less on their clinical skills such as palpation of muscles. Radiology is of course a great tool to diagnose a brain tumor and to further guide the surgeon during his therapeutic process to locate the exact area of incision. However, in many cases, the radiological findings may be misleading. The ultimate criterion for the usefulness of any diagnostic test is whether it adds crucial information beyond that otherwise available, and whether this information leads to a change in management that is ultimately beneficial to the individual patient. This is not only true for shoulder pain, but also for other clinical situations, such as neck pain and low back pain $[6,7]$.

Numerous investigations with plain radiography, myelography, computed tomography and magnetic-resonance scans of, for example, hip, neck, low back, shoulder or knee, have confirmed the high prevalence of abnormal findings in asymptomatic subjects [8-16]. There are also a lot of falsenegative results. Recent investigations in the field of rotator cuff tears have illustrated the diagnostic shortcomings of MRIa (magnetic resonance imaging with arthrography): the test is very specific but not sensitive for rotator cuff tears [17-19]. MRIa without arthrography is also highly sensitive, but has a low positive predictive value and low specificity and accuracy in detecting full-thickness rotator cuff tears [20]. The authors of this study postulated that even a total shoulder arthroplasty in patients with severe glenohumeral osteoarthritis is not always built on solid decision making. If we consider these facts along with the high percentage of false positive MRIa reports on rotator cuff tears, we also understand the concern of the authors of a 2011 study who state, "If MRIa is chosen as a diagnostic adjunct, careful consideration should be given to its interpretation to avoid unnecessary or possibly aggravating surgical intervention" [21].

The major strength of this case is that it illustrates the importance of the muscular factor in common orthopedic complaints, such as shoulder pain. The basic idea of the importance of myofascial pain was first introduced and illustrated by Travell \& Simons in their trigger point manual [22]. They describe the referred pain patterns for trigger points, and how these can be responsible for common orthopedic complaints. Muscular dysfunction is not only responsible for pain, but also for lack of strength in certain muscles and lack of myorelaxation of the affected muscles, leading to limited range of motion. According to the protocol by Travell \& Simons, injections are given at a distance from the pain zone, in the trigger points which refer the actual pain. However, the technique applied here consists of local injections in myofascial pain points (injections in the pain zone itself), not injections into trigger points which are actually at a distance from the pain zone.

In this patient, the conventional approach (intra-articular cortisone injections) produced no positive clinical outcome, and the difference between conventional diagnosis (CD) and functional diagnosis (FD) was crucial in order to have a positive outcome with the injections into the muscular pain points. In other words, the injections were not applied at the site of the enthesopathy (tuberculum majus) or at the site of the calcifications, but exclusively at the pain points in the deltoid muscle.

The limitations of this case include the lack of hard data because there is no follow-up of the ultrasound investigation, 


\section{Journal of Complementary Medicine \& Alternative Healthcare}

comparing the situation before and after treatment. Additionally, follow-up of the clinical examination of the patient before, during and after the procedure was limited as only a minimal clinical examination was performed during each session. Only the visual analog scale was used to monitor the pain of the patient.

\section{References}

1. Kersschot J (2016) Biopuncture: Definition and indications in pain management. European Journal of Integrative Medicine 8(Suppl 1): 5.

2. Kersschot J (2014) Biopuncture: The Management of Common Orthopedic and Sports Disorders. Stuttgart New York Thieme, USA, p. 45.

3. Schneider C (2011) Traumeel-an emerging option to nonsteroidal anti-inflammatory drugs in the management of acute musculoskeletal injuries. Int J Gen Med 25(4): 225-234.

4. Vanden Bossche L, Vanderstraeten G (2015) A multi-center, doubleblind, randomized, placebo-controlled trial protocol to assess Traumeel injection vs dexamethasone injection in rotator cuff syndrome: the traumeel in rotator cuff syndrome (TRARO) study protocol. BMC Musculoskelet Disord 4(16): 8.

5. Lozada C, del Rio E, Reitberg D, Smith R, Kahn C, et al. (2014) A multicenter double-blind, randomized, controlled trial (db-RCT) to evaluate the effectiveness and safety of co-administered Traumeel ${ }^{\circledR}(\operatorname{Tr} 14)$ and Zeel ${ }^{\circledR}$ (Ze14) intra-articular (IA) injections versus IA placebo in patients with moderate-to-severe pain associated with OA of the knee. Arthritis \& Rheumatology 66 (Suppl 11): S1266.

6. Andersen JC (2011) Is immediate imaging important in managing low back pain? J Athl Train 46(1): 99-102.

7. Nakashima H, Yukawa Y, Suda K, Yamagata M, Ueta T, et al. (2015) Abnormal findings on magnetic resonance images of the cervical spines in 1211 asymptomatic subjects. Spine 40(6): 392-398.

8. Abe I, Harada Y, Oinuma K, Kamikawa K, Kitahara H, et al. (2000) Acetabular labrum: abnormal findings at MR imaging in asymptomatic hips. Radiology 216(2): 576-581.

9. Fukuta S, Masaki K, Korai F (2002) Prevalence of abnormal findings in magnetic resonance images of asymptomatic knees. J Orthop Sci 7(3): 287-291.

10. Boden SD, McCowin PR, Davis DO, Dina TS, Mark AS, et al. (1990) Abnormal magnetic-resonance scans of the cervical spine in asymptomatic subjects. A prospective investigation. J Bone Joint Surg Am 72(8): 1178-1184.
11. Sher JS, Uribe JW, Posada A, Murphy BJ, Zlatkin MB (1995) Abnormal findings on magnetic resonance images of asymptomatic shoulders. J Bone Joint Surg Am 77(1): 10-15.

12. Weinreb JC, Wolbarsht LB, Cohen JM, Brown CEL, Maravilla KR (1989) Prevalence of lumbosacral intervertebral disk abnormalities on MR images in pregnant and asymptomatic nonpregnant women. Radiology 170(1 Pt 1): 125-128.

13. Englund M, Guermazi A, Gald D, Hunter DJ, Aliabadi P, et al. (2008) Incidental meniscal findings on knee MRI in middle-aged and elderly persons. N Engl J Med 359(11): 1108-1115.

14. Soder RB, Simões JD, Soder JB, Baldisserotto M (2011) MRIa of the knee joint in asymptomatic adolescent soccer players: a controlled study. AJR Am J Roentgenol 196(1): W61-W65.

15. Nakashima H, Yukawa Y, Suda K, Yamagata M, Ueta T, et al. (2015) Abnormal findings on magnetic resonance images of the cervical spines in 1211 asymptomatic subjects. Spine 40(6): 392-398.

16. Sansone V, Consonni O, Maiorano E, Meroni R, Goddi A (2015) Calcific tendinopathy of the rotator cuff: the correlation between pain and imaging features in symptomatic and asymptomatic female shoulders. Skeletal Radiol 45(1): 49-55.

17. Edmonds EW, Eisner EA, Kruk PG, Roocroft JH, Dwek JD (2015) Diagnostic shortcomings of magnetic resonance arthrography to evaluate partial rotator cuff tears in adolescents. J Pediatr Orthop 35(4): 407-411.

18. Waldt S, Bruegel M, Mueller D, Holzapfel K, Imhoff AB, et al. (2007) Rotator cuff tears: assessment with MR arthrography in 275 patients with arthroscopic correlation. Eur Radiol 17(2): 491-498.

19. Dinnes J, Loveman E, McIntyre L, Waugh N (2003) The effectiveness of diagnostic tests for the assessment of shoulder pain due to soft tissue disorders: a systematic review. Health Technol Assess 7(29): 1-166.

20. Sershon RA, Mather RC, Sherman SL, McGill KC, Romeo AA, et al. (2013) Low accuracy of interpretation of rotator cuff MRI in patients with osteoarthritis, Acta Orthop 84(5): 479-482.

21. Loeffler BJ, Brown SL, D’Alessandro DF, Fleischli JE, Connor PM (2011) Incidence of False Positive Rotator Cuff Pathology in MRIs of Patients with Adhesive Capsulitis. Orthopedics 34(5): 362.

22. Travell JG, Simons DG (1999) Myofascial Pain and Dysfunction: The Trigger Point Manual. ( $\left.2^{\text {nd }} e d n\right)$, Williams \& Wilkins, Baltimore, USA.

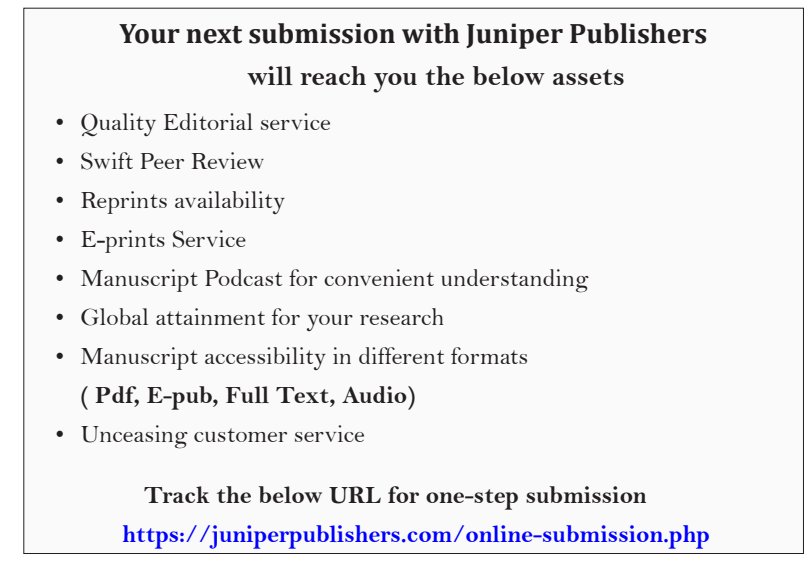

